\title{
Gradient estimates below duality exponent for a class of linear elliptic systems
}

\author{
S. Leonardi
}

Abstract. We provide sharp estimates in Lorentz spaces for the solution of the Dirichlet problem associated to the system

$$
\left\{\begin{array}{l}
A(u) \equiv-D_{i}\left(A_{i j}(x) D_{j} u\right)=f \\
u \in W_{0}^{1,1}\left(\Omega, \mathbb{R}^{N}\right)
\end{array}\right.
$$

where $\Omega$ is an open bounded subset of $\mathbb{R}^{n}(n \geq 3)$ with sufficiently regular boundary, $A(u)$ is an elliptic operator with $V M O$-coefficients and $f$ is not in the natural dual space. Moreover, when the coefficients belong to $C^{0, \alpha}(\alpha \in] 0,1[)$, we study the differentiability of the solution in BesovMorrey spaces.

Mathematics Subject Classification (2000). 35J25, 35D10.

Keywords. Elliptic systems, VMO-coefficients, $L^{1}$-data.

\section{Introduction}

In this paper we are concerned with the regularity of the (suitably defined) solution of the Dirichlet problem associated to the system ${ }^{1}$

$$
\left\{\begin{array}{l}
A(u) \equiv-D_{i}\left(A_{i j}(x) D_{j} u\right)=f \\
u \in W_{0}^{1,1}\left(\Omega, \mathbb{R}^{N}\right)
\end{array}\right.
$$

where $\Omega$ is an open bounded subset of $\mathbb{R}^{n}(n \geq 3)$ with sufficiently regular boundary, $A(u)$ is an elliptic operator with $V M O$-coefficients and $f$ is not in the natural dual space.

Namely, when the datum $f$ belongs to the Morrey space $L^{\gamma, \theta}\left(\Omega, \mathbb{R}^{N}\right)$ with

$$
\left.\left.\left.\gamma \in] 1, \frac{2 \theta}{\theta+2}\right], \quad \theta \in\right] 2, n\right]
$$

\footnotetext{
1 Einstein's convention will be used throughout the paper.
} 
we provide sharp estimates in Lorentz-Morrey spaces for the solution of the aforementioned problem while, when the coefficients belong to $C^{0, \alpha}$, for $\alpha \in$ ]0,1[, we study the differentiability of the solution in Besov-Morrey spaces.

We remark that the case $q=1$ has been investigated in the paper [12].

In the paper [19] (see also [15]) G. Mingione introduces a unified method enabling to treat simultaneously rearrangement and non-rearrangement invariant spaces. As a consequence he recovers at once all known regularity results (see $[4,5])$ about non linear elliptic equations $(N=1)$ and closes some open problems.

We will follow Mingione's approach from papers [18,19], related to nonlinear equations (see also [11]), combined with some results in [12,17], related to elliptic systems, to prove the same estimates contained in the paper by Mingione for the very weak solution of the system of linear equations (1). Moreover, we refer to the aforementioned papers for further details and remarks.

The paper is organized as follows: we start with notations; a few auxiliary results for homogeneous systems are stated in Sect. 3 ; in Sect. 4 we give a priori estimates for the weak solution of systems with regular right-hand side. In Sects. 5 and 6 we provide the integrability properties respectively of $D u$ and $u$; finally, Sect. 7 is devoted to sketch the proof of the differentiability of $D u$.

\section{Notations, functional spaces and statements of the results}

In $\mathbb{R}^{n}(n \geq 3)$, with generic point $x=\left(x_{1}, x_{2}, \ldots, x_{n}\right)$, we shall denote by $\Omega$ a bounded open nonempty set with diameter $d_{\Omega}$ and sufficiently regular boundary $\partial \Omega$.

For $R>0$ and $x^{0} \in \mathbb{R}^{n}$ we define

$$
\begin{aligned}
B_{R}\left(x^{0}\right) & =B\left(x_{o}, R\right)=\left\{x \in \mathbb{R}^{n}:\left|x-x^{0}\right|<R\right\}, \\
\Omega\left(x^{0}, R\right) & =\Omega \cap B_{R}\left(x^{0}\right), \\
Q_{R}\left(x^{0}\right) & =\left\{x \in \mathbb{R}^{n}: \sup _{1 \leq i \leq n}\left|x_{i}-x_{i}^{0}\right|<R\right\}, \\
d\left(x^{0}, \partial \Omega\right) & =\operatorname{dist}\left(x^{0}, \partial \Omega\right) .
\end{aligned}
$$

We shall often use the short notation $B_{R}$ and $Q_{R}$ instead of $B_{R}\left(x^{0}\right)$ and $Q_{R}\left(x^{0}\right)$ respectively, when no ambiguity will arise.

Moreover, if $u \in L^{1}\left(B, \mathbb{R}^{N}\right)$ and $0<|B|<+\infty^{2}$ we denote by

$$
u_{B}:=\frac{1}{|B|} \int_{B} u(x) d x .
$$

Let us define the functional spaces we will use. In order to simply the exposition we adopt a slight modification of the usual definitions of function spaces we deal with.

\footnotetext{
${ }^{2}|B|$ is the $n$-dimensional Lebesgue measure of $B$.
} 
Definition 2.1. (Morrey space) Let $q \geq 1$ and $\theta \in[0, n]$. By $L^{q, \theta}\left(\Omega, \mathbb{R}^{N}\right)$ we denote the space of all vector-functions $u \in L^{q}\left(\Omega, \mathbb{R}^{N}\right)$ such that

$$
\|u\|_{L^{q, \theta}(\Omega)}=\sup _{B_{R} \subseteq \Omega, R \leq 1}\left\{R^{\theta-n} \int_{B_{R}}|u(x)|^{q} d x\right\}^{1 / q}
$$

is finite. $L^{q, \theta}\left(\Omega, \mathbb{R}^{N}\right)$ equipped with the above norm is a Banach space.

Definition 2.2. (Campanato space) Let $q \geq 1$ and $0 \leq \lambda<n+q$. By $\mathcal{L}^{q, \lambda}\left(\Omega, \mathbb{R}^{N}\right)$ we denote the space of all vector-functions $u \in L^{q}\left(\Omega, \mathbb{R}^{N}\right)$ such that

$$
[u]_{\mathcal{L}^{q, \lambda}(\Omega)}=\sup _{x^{0} \in \Omega, 0<\rho \leq d_{\Omega}}\left\{\rho^{-\lambda} \int_{\Omega\left(x^{0}, \rho\right)}\left|u(x)-u_{\Omega\left(x^{o}, \rho\right)}\right|^{q} d x\right\}^{1 / q}<+\infty .
$$

Now, starting from the usual definition of Lorentz, Marcinkiewicz, Orlicz and fractional Sobolev spaces, respectively denoted by $L(q, s)\left(\Omega, \mathbb{R}^{N}\right), \mathcal{M}^{q}$ $\left(\Omega, \mathbb{R}^{N}\right), L \log L\left(\Omega, \mathbb{R}^{N}\right)$ and $W^{\eta, q}\left(\Omega, \mathbb{R}^{N}\right)$, we define their obvious "Morrey -like" extension.

Definition 2.3. (Lorentz-Morrey space) Let $q \geq 1, s>0$ and $\theta \in[0, n]$. By $L^{\theta}(q, s)\left(\Omega, \mathbb{R}^{N}\right)$ we denote the space of all vector-functions $u: \Omega \rightarrow \mathbb{R}^{N}$ such that the quantity

$$
\sup _{B_{R} \subseteq \Omega, R \leq 1} R^{\frac{\theta-n}{q}}\|u\|_{L(q, s)\left(B_{R}\right)}<+\infty
$$

where

$$
\|u\|_{L(q, s)\left(B_{R}\right)}=\left(q \int_{0}^{+\infty}\left(\lambda^{q}\left|\left\{x \in B_{R}:|u(x)|>\lambda\right\}\right|\right)^{\frac{s}{q}} \frac{d \lambda}{\lambda}\right)^{1 / s} .
$$

We denote by

$$
\|u\|_{L^{\theta}(q, s)(\Omega)}=\|u\|_{L(q, s)(\Omega)}+\sup _{B_{R} \subset \Omega, R \leq 1} R^{\frac{\theta-n}{q}}\|u\|_{L(q, s)(\Omega)} .
$$

Definition 2.4. (Marcinkiewicz-Morrey space) We denote by $\mathcal{M}^{q, \theta}\left(\Omega, \mathbb{R}^{N}\right), q \geq$ $1, \theta \in[0, n]$, the space of all vector-functions $u: \Omega \rightarrow \mathbb{R}^{N}$ such that

$$
\sup _{B_{R} \subseteq \Omega, R \leq 1} R^{\frac{\theta-n}{q}}\|u\|_{\mathcal{M}^{q}\left(B_{R}\right)}<+\infty
$$

where

$$
\|u\|_{\mathcal{M}^{q}\left(B_{R}\right)}=\sup _{\lambda>0} \lambda\left|\left\{x \in B_{R}:|u(x)|>\lambda\right\}\right|^{1 / q} .
$$

We denote by

$$
\|u\|_{\mathcal{M}^{q, \theta}(\Omega)}=\|u\|_{\mathcal{M}^{q}(\Omega)}+\sup _{B_{R} \subseteq \Omega, R \leq 1} R^{\frac{\theta-n}{q}}\|u\|_{\mathcal{M}^{q}\left(B_{R}\right)} .
$$

Definition 2.5. (Orlicz-Morrey space) We denote by $L^{\theta} \log L\left(\Omega, \mathbb{R}^{N}\right), \theta \in$ $[0, n]$, the space of all measurable vector-functions $u: \Omega \rightarrow \mathbb{R}^{N}$ such that the quantity

$$
\|u\|_{L^{\theta} \log L(\Omega)}=\sup _{B_{R} \subseteq \Omega, R \leq 1} R^{\theta-n}\|u\|_{L \log L\left(B_{R}\right)}<+\infty
$$


where

$$
\|u\|_{L \log L\left(B_{R}\right)}=\inf \left\{\lambda>0: \int_{B_{R}}\left|\frac{u}{\lambda}\right| \log \left(e+\left|\frac{u}{\lambda}\right|\right) d x \leq 1\right\} .
$$
$[6,7])$.

Finally, let us define the Sobolev-Morrey spaces of fractional order (see

Definition 2.6. (Fractional Sobolev-Morrey space) For fixed $\eta \in] 0,1], q \geq 1$ and $\theta \in[0, n]$. We denote by $W^{\eta, q, \theta}\left(\Omega, \mathbb{R}^{N}\right)$ the space of all vector functions $u: \Omega \rightarrow \mathbb{R}^{N}$ such that the following quantity

$$
[u]_{\eta, q, \theta, \Omega}^{q}=\left\{\begin{array}{ll}
\|D u\|_{L^{q, \theta}(\Omega)}^{q} & \text { if } \eta=1 \\
\sup _{B_{R} \subseteq \Omega, R \leq 1} R^{\theta-n}[u]_{\eta, q, B_{R}}^{q} & \text { if } \eta<1
\end{array}<+\infty\right.
$$

where

$$
[u]_{\eta, q, B_{R}}=\left(\int_{B_{R}} \int_{B_{R}} \frac{|u(x)-u(y)|^{q}}{|x-y|^{n+\eta q}} d x d y\right)^{\frac{1}{q}} .
$$

$W^{\eta, q, \theta}\left(\Omega, \mathbb{R}^{N}\right)$ equipped with the norm

$$
\|u\|_{W^{\eta, q, \theta}(\Omega)}=\|u\|_{L^{q}(\Omega)}+[u]_{\eta, q, \Omega}+[u]_{\eta, q, \theta, \Omega}
$$

is a Banach space.

Moreover we introduce the notion of BMO and VMO classes.

Definition 2.7. (John-Nirenberg space) Let $Q$ be a cube in $\mathbb{R}^{n}$. By $\operatorname{BMO}(Q)$ we denote the space of all functions $u \in L^{1}\left(Q, \mathbb{R}^{N^{2}}\right)$ such that the seminorm defined by

$$
[u]_{B M O(Q)}=\sup _{\tilde{Q} \subset Q} \frac{1}{|\tilde{Q}|} \int_{|\tilde{Q}|}\left|u-u_{\tilde{Q}}\right| d x
$$

is finite, where the supremum is taken over all cubes with sides parallel to coordinate axes.

Let us recall that $\mathcal{L}^{q, n}(Q) \cong \operatorname{BMO}(Q), \forall q \geq 1$.

Definition 2.8. (Sarason space) For a matrix-function $w \in L^{1}\left(\Omega, \mathbb{R}^{N^{2}}\right)$ and $r>0$ we define

$$
V(x, r) \equiv \sup _{0<\rho \leq r} \frac{1}{|\Omega(x, \rho)|} \int_{\Omega(x, \rho)}\left|w(y)-w_{\Omega(x, \rho)}\right| d y
$$

and we introduce the $V M O$-continuity modulus for $w$

$$
V(r) \equiv \sup _{x \in \Omega} V(x, r) .
$$

By $V M O$ we denote the space of all matrix-functions $w \in L^{1}\left(\Omega, \mathbb{R}^{N^{2}}\right)$ such that

$$
V(r)<+\infty \text { for all } 0<r \leq d_{\Omega}
$$

and

$$
\lim _{r \rightarrow 0} V(r)=0 .
$$


If $u: \Omega \rightarrow \mathbb{R}^{N}$, we set

$$
D_{i} \equiv \frac{\partial}{\partial x_{i}}, \quad D u=\left(D_{i} u^{r}\right)_{\substack{i=1, \ldots, n \\ r=1, \ldots, N}} .
$$

Let $A_{i j}(x)=\left(A_{i j}^{r s}(x)\right)_{r, s=1, \ldots, N}, i, j=1,2, \ldots, n$, be matrix-functions for which the following conditions are satisfied:

there exist two positive constants $\Lambda_{1}$ and $\Lambda_{2}$ such that

$$
\begin{gathered}
\Lambda_{2} \geq 1 \geq \Lambda_{1}, \\
\qquad \begin{array}{c}
\Lambda_{2}|\xi|^{2} \geq A_{i j}(x) \xi_{i} \xi_{j} \geq \Lambda_{1}|\xi|^{2} \\
\text { for a.a. } x \in \Omega, \quad \forall \xi_{i}=\left(\xi_{i}^{r}\right) \in \mathbb{R}^{N}, \quad i=1,2, \ldots, n, \\
A_{i j}^{r s}(x)=A_{j i}^{s r}(x) \quad i, j=1, \ldots, n ; r, s=1, \ldots, N \\
\text { for a.a. } \quad x \in \Omega
\end{array}
\end{gathered}
$$

and

$$
A_{i j}(x) \in L^{\infty}\left(\Omega, \mathbb{R}^{N^{2}}\right) \cap V M O, \quad i, j=1,2, \ldots, n .
$$

Assume moreover that

$$
\mu \in M_{b}\left(\Omega, \mathbb{R}^{N}\right),
$$

where $M_{b}\left(\Omega, \mathbb{R}^{N}\right)$ denotes the space of the Radon vector-measures with finite total variation $|\mu|(\Omega) \mid<+\infty$, and let us consider the following Dirichlet problem

$$
\begin{cases}A(u) \equiv-D_{i}\left(A_{i j}(x) D_{j} u\right)=\mu & \text { in } \Omega \\ u=0 & \text { on } \partial \Omega .\end{cases}
$$

Let us first recall that the VMO space of functions with "vanishing mean oscillations", introduced by Sarason in [20], turns out to be very useful in the study of smoothness of weak solutions to elliptic equations or systems (see [9] for a survey and [2]). In fact the VMO condition provides the natural integral-type generalization of continuity allowing for extending several classical results for constant coefficients problems to those with variable ones.

Due to a celebrated De Giorgi's counterexample [14], it is well known that the elliptic systems with coefficients only measurable and bounded need not have continuous solutions for $n \geq 3$; while an extra structural condition like VMO or Cordes type guarantees the Hölder continuity of the solution (see [2] or [16]).

For the problem (6) we shall adopt the following notion of solution.

Definition 2.9. We say that a vector-function $u \in W_{0}^{1,1}\left(\Omega, \mathbb{R}^{N}\right)$ is a very weak solution (briefly a Stampacchia solution) of the system (6) if it satisfies

$$
\begin{aligned}
& \int_{\Omega} u A(\varphi) d x=\int_{\Omega} \varphi d \mu, \\
& \quad \forall \varphi \in\left\{\varphi \in W_{0}^{1,2}\left(\Omega, \mathbb{R}^{N}\right) \cap C^{0}\left(\bar{\Omega}, \mathbb{R}^{N}\right): A(\varphi) \in C^{0}\left(\bar{\Omega}, \mathbb{R}^{N}\right)\right\} .
\end{aligned}
$$

The existence and uniqueness of such a solution, whenever $\mu \in L^{1}\left(\Omega, \mathbb{R}^{N}\right)$, has been proved in [16] (see also [13]), provided the matrix $\left(A_{i j}\right)$ has bounded entries $A_{i j}$ and sufficiently small dispersion of the eigenvalues (i.e. a Cordes 
condition), and in [17] provided $A_{i j}$ belong to $L^{\infty}\left(\Omega, \mathbb{R}^{N^{2}}\right) \cap V M O$. The proof remains unchanged whenever the right hand side belongs to $M_{b}\left(\Omega, \mathbb{R}^{N}\right)$.

Moreover, in $[13,16,17]$ it was proven also that $u \in W_{0}^{1, q}\left(\Omega, \mathbb{R}^{n N}\right)$ for any $q \in\left[1, \frac{n}{n-1}[\right.$.

Here we shall start assuming that $\mu \in L^{\gamma}$ where the range of the exponent $\gamma$ of interest is the one dominated by the duality exponent, i.e. $\gamma$ is such that $L^{\gamma} \nsubseteq W^{-1,2} \equiv\left(W^{1,2}\right)^{*}$, so that one initially considers

$$
1<\gamma \leq \frac{2 n}{n+2} \equiv\left(2^{*}\right)^{\prime}
$$

Analogously, when considering the Morrey space $L^{\gamma, \theta}$ we always assume that the parameters $\gamma$ and $\theta$ are such that

$$
2 \leq \theta \leq n, \quad 1 \leq \gamma \leq \frac{2 \theta}{\theta+2} \leq\left(2^{*}\right)^{\prime}
$$

since it is well known that $L^{1, \theta} \subset W^{-1,2}$ for $\theta<2$ (see $[3,21]$ and the appendix of [10]) and in this case some regularity results con be found e.g. in [17].

Here we can prove the following

Theorem 2.1. Let $\Omega$ be a bounded domain with $C^{2}$-boundary and $f \in L^{\gamma}$ $\left(\Omega, \mathbb{R}^{N}\right)$, with $\left.\left.\gamma \in\right] 1, \frac{2 n}{n+2}\right]$. Let conditions (3) and (4) be satisfied.

Then the Stampacchia solution $u \in W_{0}^{1,1}\left(\mathbb{R}^{n N}\right)$ of the problem (1) belongs to $W_{0}^{1, q}\left(\Omega, \mathbb{R}^{N}\right)$ for any $q \in\left[1, \frac{n \gamma}{n-\gamma}\right]$.

Moreover, there exists a positive constant $c=c_{V}\left(n, q, \Lambda_{1}, \Lambda_{2}, \Omega\right)^{3}$ such that

$$
\|u\|_{W_{0}^{1, q}(\Omega)} \leq c\|f\|_{L^{\gamma}(\Omega)} .
$$

Proof. If $A$ satisfies (3) then, by Lax-Milgram theorem, there exists a linear continuous operator $G: W^{-1,2}\left(\Omega, \mathbb{R}^{N}\right) \rightarrow W_{0}^{1,2}\left(\Omega, \mathbb{R}^{N}\right)$ such that $\tilde{u}=G(T)$ is the unique weak solution of the equation

$$
A(\tilde{u})=T \text {. }
$$

For $p \in\left[2, n\left[\right.\right.$ consider $T=D_{i} g_{i}$, with $g_{i} \in L^{p}$, and $A_{i j}$ satisfying (3) and (4). Then by Theorem 3.4 of [2] we have

$$
\|D \tilde{u}\|_{L^{p}(\Omega)} \leq c_{V}\left(n, p, \Lambda_{1}, \Lambda_{2}, \Omega\right)\|g\|_{L^{p}(\Omega)} .
$$

Thus, Sobolev embedding Theorem yields

$$
\|\tilde{u}\|_{L^{p^{*}}(\Omega)} \leq c_{V}\left(n, p, \Lambda_{1}, \Lambda_{2}, \Omega\right)\|g\|_{L^{p}(\Omega)} .
$$

As the inequality (10) holds for any representation $T=D_{i} g_{i}$ we have

$$
\|\tilde{u}\|_{L^{p^{*}}(\Omega)} \leq c_{V}\left(n, p, \Lambda_{1}, \Lambda_{2}, \Omega\right)\|T\|_{W^{-1, p}(\Omega)} .
$$

Thus $G$ maps continuously $W^{-1, p}\left(\Omega, \mathbb{R}^{N}\right)$ into $L^{p^{*}}\left(\Omega, \mathbb{R}^{N}\right)$.

\footnotetext{
3 As a permanent convention we will denote by $c_{V}(\ldots, \Omega)$ a constant which depends on various parameters, on the coefficients of the system through the smallness of their VMOcontinuity modulus and on the geometrical properties of the involved domain $\Omega$.
} 
On the other hand (7) holds if and only if

$$
\int_{\Omega} u \psi d x=\int_{\Omega} f G(\psi) d x, \quad \forall \psi \in C^{0}\left(\bar{\Omega}, \mathbb{R}^{N}\right)
$$

i.e. if and only if $u=G^{*}(f)$ for $G^{*}$ adjoint of $G$.

Since $G$ maps continuously $W^{-1, p}\left(\Omega, \mathbb{R}^{N}\right)$ into $L^{p^{*}}\left(\Omega, \mathbb{R}^{N}\right)$, then $G^{*}$ is a continuous linear operator from $L^{{p^{*}}^{\prime}}\left(\Omega, \mathbb{R}^{N}\right)$ into $W_{0}^{1, p^{\prime}}\left(\Omega, \mathbb{R}^{N}\right)$, with $\frac{1}{p}+\frac{1}{p^{\prime}}=$ $1,{ }^{4}$ and $\left\|G^{*}\right\| \leq\|G\|$ and this implies the thesis.

Corollary 2.1. Let $\Omega=B_{R}\left(x^{0}\right)$, with $0<R \leq 1$, and let the assumptions of the Theorem be satisfied.

Then there exists a unique Stampacchia solution $u$ of the problem (1) such that $u \in W_{0}^{1, q}\left(\Omega, \mathbb{R}^{N}\right)$ for any $q \in\left[1, \frac{n \gamma}{n-\gamma}\right]$.

Moreover, there exists a positive constant $c=c_{V}\left(n, q, \Lambda_{1}, \Lambda_{2}\right)$ such that

$$
\|u\|_{W_{0}^{1, q}(\Omega)} \leq c R^{1-n\left(\frac{1}{\gamma}-\frac{1}{q}\right)}\|f\|_{L \gamma(\Omega)} .
$$

Proof. By the previous Theorem, the Corollary is true for $\Omega=B_{1}(0)$ and (13) follows directly from $(8)$.

To get (13) for $R<1$, let us perform the following change of variables

$$
\begin{aligned}
& \tilde{u}(y):=R^{-1} u\left(x_{o}+R y\right), \quad \tilde{A}_{i j}(y):=A_{i j}\left(x_{o}+R y\right), \\
& \tilde{f}(y):=R f\left(x_{o}+R y\right), \quad y \in B_{1}(0) .
\end{aligned}
$$

and note that the transformed coefficients $\tilde{A}_{i j}$ are still in VMO class.

In fact it is not difficult to see that, denoted by $\tilde{V}$ the $V M O$-continuity modulus for $\tilde{A}_{i j}$, it is

$$
\tilde{V}(r)=V(R r) \leq V(r) .
$$

As a consequence, it holds (8) for $\tilde{u}$ and thus a change back of variables completes the proof.

\section{Auxiliary results}

In this section we state and prove some regularity results for weak solutions to homogeneous elliptic systems.

The next lemma deals with the solutions to homogeneous systems with VMO-coefficients.

Lemma 3.1. Let assumptions (3) and (4) be satisfied, let $q \in[1,2]$ and let $v \in W^{1,2}\left(\Omega, \mathbb{R}^{N}\right)$ be a weak solution to the system

$$
-D_{i}\left(A_{i j}(x) D_{j} v\right)=0 \quad \text { in } \Omega .
$$

\footnotetext{
${ }^{4}$ Observe that if $p \in\left[2, n\left[\right.\right.$ then $\left.\left.\left(p^{*}\right)^{\prime} \equiv \gamma \in\right] 1, \frac{2 n}{n+2}\right]$ and that $p^{\prime}=\frac{n \gamma}{n-\gamma}$.
} 
Then, there exist three positive constants $\beta=\beta(n) \in] 0,1 / 2], c=c\left(n, q, \frac{\Lambda_{1}}{\Lambda_{2}}\right)$ and $\rho_{0}=\rho_{V}\left(n, q, \frac{\Lambda_{1}}{\Lambda_{2}}\right)$ such that it holds

$$
\int_{B_{\rho}}|D v|^{q} d x \leq c\left(\frac{\rho}{R}\right)^{n-q+\beta q} \int_{B_{R}}|D v|^{q} d x
$$

and

$$
\int_{B_{\rho}}|v|^{q} d x \leq c\left(\frac{\rho}{R}\right)^{n} \int_{B_{R}}|v|^{q} d x
$$

for every $B_{R} \subset \subset \Omega$ with $R<\rho_{0}$ and $\left.\left.\rho \in\right] 0, R\right]$.

Moreover, there exist $\chi=\chi\left(n, \frac{\Lambda_{1}}{\Lambda_{2}}\right)>1$ and $c=c\left(n, q, \frac{\Lambda_{1}}{\Lambda_{2}}\right)>0$ such that

$$
D v \in L_{l o c}^{2 \chi}\left(\Omega, \mathbb{R}^{n N}\right)
$$

and

$$
\left(f_{B_{\frac{R}{2}}}|D v|^{2 \chi} d x\right)^{\frac{1}{2 \chi}} \leq c\left(f_{B_{R}}|D v|^{q} d x\right)^{\frac{1}{q}},{ }^{5}
$$

holds for any $B_{R} \subset \subset \Omega$, while, for every $\chi_{0}>1$, it holds that

$$
\left(f_{B_{\frac{R}{2}}}|v|^{2 \chi_{0}} d x\right)^{\frac{1}{2 \chi_{0}}} \leq c\left(f_{B_{R}}|v|^{q} d x\right)^{\frac{1}{q}} .
$$

Proof. The estimates (15) and (18) have been proved respectively in Theorem 5.1 of [17] and in Lemma 3.2 of [12].

As far as it concerns estimates (16) and (19) let us observe that they readily follow from inequality (63) of [17].

Indeed, if $\rho<R / 2^{6}$ we have

$$
\begin{aligned}
\int_{B_{\rho}}|v|^{q} d x & \leq c_{1}\left(n, \Lambda_{1}, \Lambda_{2}\right) \rho^{n} \sup _{B_{R / 2}}|v|^{q} \\
& \leq c_{1} \rho^{n} R^{-n} \int_{B_{R}}|v|^{q} d x
\end{aligned}
$$

which is (16). Analogously, for any $\chi_{0}>1$, we estimate

$$
\int_{B_{\frac{R}{2}}}|v|^{2 \chi_{0}} d x \leq c_{1}\left[R^{-n} \int_{B_{R}}|v|^{q} d x\right]^{\frac{2 \chi_{0}-q}{q}} \int_{B_{R}}|v|^{q} d x
$$

which is (19).

\footnotetext{
5 This inequality holds without any restriction on $R$ as it can be proven under the weaker assumption $A_{i j} \in L^{\infty}\left(\Omega, \mathbb{R}^{N^{2}}\right)$.

${ }^{6}$ The case $\rho \geq R / 2$ being obvious.
} 


\section{Systems with regular right-hand side}

In this section we establish some a priori estimates for the weak solution of the Dirichlet problem related to systems with regular right-hand sides.

Namely, from now on we shall suppose $f \in L^{\infty}\left(\Omega, \mathbb{R}^{N}\right)$ and we let $u \in$ $W_{0}^{1,2}\left(\Omega, \mathbb{R}^{N}\right)$ be the weak solution to the Dirichlet problem

$$
\begin{cases}-D_{i}\left(A_{i j}(x) D_{j} u\right)=f & \text { in } \Omega \\ u=0 & \text { on } \partial \Omega\end{cases}
$$

under the structural assumptions (3) and (4).

Moreover, for any $x^{0} \in \Omega$ let us fix a ball $B_{R}=B_{R}\left(x^{0}\right) \subset \subset \Omega$ and let us consider the weak solution $v \in W^{1,2}\left(B_{R}, \mathbb{R}^{N}\right)$ to the following Dirichlet problem

$$
\begin{cases}-D_{i}\left(A_{i j}(x) D_{j} v\right)=0 & \text { in } B_{R} \\ v=u & \text { on } \partial B_{R} .\end{cases}
$$

Thus we can prove the next

Lemma 4.1. Let $f \in L^{\theta}(\gamma, s)\left(B_{R}, \mathbb{R}^{N}\right)$, with $R \leq 1$, for some $\gamma>1$ and $s \in] 0,+\infty]$, and let $u \in W_{0}^{1,2}\left(\Omega, \mathbb{R}^{N}\right)$ and $v \in W^{1,2}\left(B_{R}, \mathbb{R}^{N}\right)$ respectively solutions of the problems (20) and (21).

Then there exists a positive constant $c=c\left(n, \gamma, \Lambda_{1}, \Lambda_{2}\right)$ such that

$$
\int_{B_{R}}\left(R^{-1}|u-v|+|D u-D v|\right) d x \leq c R^{n-\frac{\theta-\gamma}{\gamma}}\|f\|_{L^{\theta}(\gamma, s)\left(B_{R}\right)} .
$$

Proof. The proof follows the lines of Lemma 9 from [19].

Namely, we start from (16) of Lemma 4.1 of [12] i.e.

$$
\int_{B_{R}}|D u-D v| d x \leq c R \int_{B_{R}}|f| d x
$$

then we use Poincaré inequality (see e.g. [1]) and inequalities (4.12) and (4.16) of [19] to deduce the thesis.

Let $Q_{0}$ be a cube such that $\left|Q_{0}\right| \leq 1$ and such that the magnified cube $n^{2} Q_{0} \subset \subset \Omega{ }^{7}$ For a given function $g \in L^{1}$, we denote by

$$
\begin{aligned}
& M^{*}(g)(x)=\sup _{Q \subseteq n^{2} Q_{0}, x \in Q} f_{Q}|g(y)| d y, \\
& M_{\beta}^{*}(g)(x)=\sup _{Q \subseteq n^{2} Q_{0}, x \in Q}|Q|^{\frac{\beta}{n}} f_{Q}|g(y)| d y
\end{aligned}
$$

where the supremum is taken over the cubes $Q$ with sides parallel to those of $Q_{0}$.

Following the proof of Lemma 10 by Mingione [19] we can prove the following

\footnotetext{
$7 n^{2} Q_{0}$ is the cube with side length $n^{2}$-times the side length of $Q_{0}$.
} 
Lemma 4.2. Let $u \in W_{0}^{1,2}\left(\Omega, \mathbb{R}^{N}\right)$ be the solution to the problem (20). Then, for every $T>1$ there exists a number $\left.\varepsilon=\varepsilon\left(n, \Lambda_{1}, \Lambda_{2}, T\right) \in\right] 0,1[$, such that if $\lambda>0$ and $Q \subset Q_{0}$ is a dyadic sub-cube of $Q_{0}$ such that

$$
\left|Q \cap\left\{x \in Q_{0}: M^{*}(|D u|)(x)>C T \lambda, M_{1}^{*}(|f|)(x) \leq \varepsilon \lambda\right\}\right|>T^{-2 \chi}|Q|,
$$

then it predecessor $\tilde{Q}$ satisfies

$$
\tilde{Q} \subseteq\left\{x \in Q_{0}: M^{*}(|D u|)(x)>\lambda\right\} .
$$

Here $\chi=\chi\left(n, \Lambda_{1}, \Lambda_{2}\right)>1$ is the higher integrability exponent introduced in Lemma 3.1, while $C=C\left(n, \Lambda_{1}, \Lambda_{2}\right)>1$ is an absolute constant.

Proof. We assume, by contradiction, that (25) is false. Then, arguing as in [19], we can prove that

$$
f_{3 Q}|D u| d x \leq \lambda
$$

and that

$$
M_{1}^{*}(|f|)(\bar{x}) \leq \varepsilon \lambda
$$

for some $\bar{x} \in Q$.

Now, we let $B_{R}$, with $R \leq 1$, be the ball having the cube $3 Q$ as inner cube $^{8}$ and such that $B_{R} \subset n^{2} Q_{0}$. In $B_{R}$ we consider the Dirichlet problem

$$
\left\{\begin{array}{l}
-D_{i}\left(A_{i j}(x) D_{j} v\right)=0 \\
v-u \in W_{0}^{1,2}\left(B_{R}\right)
\end{array}\right.
$$

Observing that

$$
\left|B_{R}\right|^{\frac{1}{n}} f_{B_{R}}|f| d x \leq c(n) \varepsilon \lambda
$$

and exploiting Lemma 4.1 from [12] we deduce

$$
f_{3 Q}|D u-D v| d x \leq c_{V}\left(n, \Lambda_{1}, \Lambda_{2}\right) \varepsilon \lambda .
$$

As far as it concerns $v$, from (18) we deduce

$$
\left(f_{2 Q}|D v|^{2 \chi} d x\right)^{\frac{1}{2 \chi}} \leq c\left(n, \Lambda_{1}, \Lambda_{2}\right) f_{3 Q}|D v| d x
$$

where $\chi$ is the number introduced in Lemma 3.1.

On the other hand, by (26) and (30) we get

$$
f_{3 Q}|D v| d x \leq c f_{3 Q}|D u| d x+c f_{3 Q}|D u-D v| d x \leq c \lambda
$$

and this last inequality together with (31) give

$$
\int_{2 Q}|D v|^{2 \chi} d x \leq c \lambda^{2 \chi}
$$

\footnotetext{
${ }^{8}$ We shall call inner cube of a ball $B$ the largest cube, concentric to $B$ and with sides parallel to the coordinate axes, contained in $B$. The inner cube of $B$ will be denoted by $Q_{i n n}(B)$.
} 
The aforementioned inequality corresponds to the inequality (6.13) of [19] and so the proof can be completed as in Lemma 10 of [19].

Lemma 4.3. Let $u \in W_{0}^{1,2}\left(\Omega, \mathbb{R}^{N}\right)$ be the solution of the problem (20) and let $f \in L^{\theta}(\gamma, s)\left(\Omega, \mathbb{R}^{N}\right)$ with $1<2 \gamma \leq \theta \leq n$ and $\left.\left.s \in\right] 0,+\infty\right]$. Then there exists a positive constant $c=c_{V}\left(n, \Lambda_{1}, \Lambda_{2}, \gamma, s\right)$ such that

$$
\|D u\|_{L^{1, \frac{\theta-\gamma}{\gamma}\left(B_{t}\right)}} \leq c\left[(d-t)^{\frac{\theta-\gamma}{\gamma}-n}\|D u\|_{L^{1}\left(B_{d}\right)}+\|f\|_{L^{\theta}(\gamma, s)\left(B_{d}\right)}\right.
$$

holds for every couple of concentric balls $B_{t} \subset B_{d} \subset \subset \Omega$.

Proof. Let us fix $x_{0} \in B_{t}$ and a ball $B_{R}\left(x_{0}\right), 0<R \leq \min \left\{1, \rho_{0}, d\left(x_{0}, \partial B_{d}\right)\right\},{ }^{9}$ such that $B_{R} \subseteq B_{d}$.

Then, for the solution $v$ of the problem (21) we have the estimate (15) with $q=1$, i.e.

$$
\int_{B_{\rho}}|D v| d x \leq c\left(\frac{\rho}{R}\right)^{n-1+\beta} \int_{B_{R}}|D v| d x
$$

for any $\rho \in] 0, R[$.

From the aforementioned inequality we deduce

$$
\begin{aligned}
\int_{B_{\rho}}|D u| d x & \leq c\left(\frac{\rho}{R}\right)^{n-1+\beta} \int_{B_{R}}|D v| d x+c \int_{B_{R}}|D v-D u| d x \\
& \leq c\left(\frac{\rho}{R}\right)^{n-1+\beta} \int_{B_{R}}|D u| d x+c \int_{B_{R}}|D v-D u| d x .
\end{aligned}
$$

The last integral can be estimated using Lemma 4.1 and thus, by the mean of (35), we get

$$
\int_{B_{\rho}}|D u| d x \leq c\left(\frac{\rho}{R}\right)^{n-1+\beta} \int_{B_{R}}|D u| d x+c R^{n-\frac{\theta-\gamma}{\gamma}}\|f\|_{L^{\theta}(\gamma, s)\left(B_{d}\right)} .
$$

An algebraic lemma by Campanato (see e.g. [8, Chap. 1]) allows us to conclude with

$$
\int_{B_{\rho}}|D u| d x \leq c\left[(d-t)^{\frac{\theta-\gamma}{\gamma}-n} \int_{B_{d}}|D u| d x+\|f\|_{L^{\theta}(\gamma, s)\left(B_{d}\right)}\right] \rho^{n-\frac{\theta-\gamma}{\gamma}}
$$

where $c=c_{V}\left(n, \Lambda_{1}, \Lambda_{2}, \gamma, s\right)$.

The aforementioned inequality and a covering argument similar to the one in Corollary 3.2 of [17] concludes the proof.

\section{Systems with right-hand side not in the natural dual space}

In this Section we suppose again that the conditions (3) and (4) be satisfied.

Following the proof in [19, p. 611], we are now in the position to prove the following fundamental

\footnotetext{
${ }^{9} \rho_{0}$ is the number which occurred in the the Lemma 3.1.
} 
Theorem 5.1. Let $f \in L^{\theta}(\gamma, s)\left(\Omega, \mathbb{R}^{N}\right)$, with $\left.\left.\left.\left.\left.\left.\gamma \in\right] 1, \frac{2 \theta}{\theta+2}\right], \theta \in\right] 2, n\right], s \in\right] 0,+\infty\right]$, and let $u \in W_{0}^{1,1}\left(\Omega, \mathbb{R}^{N}\right)$ be the Stampacchia solution to the problem (20). Then

$$
D u \in L_{l o c}^{\theta}\left(\frac{\theta \gamma}{\theta-\gamma}, \frac{s \theta}{\theta-\gamma}\right)\left(\Omega, \mathbb{R}^{n N}\right)
$$

and there exists a positive constant $c=c\left(n, \Lambda_{1}, \Lambda_{2}, \gamma, s\right)$ such that the estimate

$$
\|D u\|_{L^{\theta}\left(\frac{\theta \gamma}{\theta-\gamma}, \frac{s \theta}{\theta-\gamma}\right)\left(B_{R / 2}\right)} \leq c R^{\frac{\theta-\gamma}{\gamma}-n}\left[\|D u\|_{L^{1}\left(B_{R}\right)}+\|f\|_{L^{\theta}(\gamma, s)\left(B_{R}\right)}\right]
$$

holds for every ball $B_{R} \subset \subset \Omega$.

Proof. We will argue as in the Step 5 of Theorem 11 from [19].

Let $\left\{f_{k}\right\}$ be a sequence of $L^{\infty}$-regular functions such that

$$
f_{k} \rightarrow f \quad \text { strongly in } L^{\gamma}\left(\Omega, \mathbb{R}^{N}\right)
$$

and

$$
\left\|f_{k}\right\|_{L^{\gamma}(\Omega)} \leq\|f\|_{L^{\gamma}(\Omega)} \quad \forall k \in \mathbb{N} .
$$

For any $k \in \mathbb{N}$, let us consider the unique weak solution $u_{k} \in W_{0}^{1,2}\left(\Omega, \mathbb{R}^{N}\right)$ of the problem (20) with $f=f_{k}$ and observe that $u_{k}$ is also the unique Stampacchia solution of the same problem.

Now, we fix a ball $B_{\rho} \subset \subset \Omega$, with $\left.\left.\rho \in\right] 0,1\right]$, and we consider the problem (20) in $B_{\rho}$. In view of the standard rescaling procedure used in the Corollary 2.1 we switch to $\tilde{u}$ and $\tilde{f}$ defined on $B_{1}$.

Proceeding as in the Steps 2 and 3 of the aforementioned paper ${ }^{10}$ one can prove the following inequality

$$
\left\|D \tilde{u}_{k}\right\|_{L\left(\frac{\theta \gamma}{\theta-\gamma}, \frac{\theta s}{\theta-\gamma}\right)(\tilde{Q})} \leq c\left[\left(f_{n^{2} \tilde{Q}}\left|D \tilde{u}_{k}\right| d x\right)|\tilde{Q}|^{\frac{\theta-\gamma}{\theta \gamma}}+\left\|\tilde{f}_{k}\right\|_{L^{\theta}(\gamma, s)\left(n^{2} \tilde{Q}\right)}\right]
$$

with $c=c_{V}\left(n, \Lambda_{1}, \Lambda_{2}, \gamma, s\right)$ and $\tilde{Q} \equiv Q_{i n n}\left(B_{1}\right) \cdot{ }^{11}$

Passing to inner and outer balls of $\tilde{Q}$ and applying inequality (40) we obtain

$$
\left\|D \tilde{u}_{k}\right\|_{L\left(\frac{\theta \gamma}{\theta-\gamma}, \frac{\theta s}{\theta-\gamma}\right)\left(B_{1 / n^{2}}\right)} \leq c\left[\left\|D \tilde{u}_{k}\right\|_{L^{1, \frac{\theta-\gamma}{\gamma}\left(B_{9 / 10}\right)}}+\left\|\tilde{f}_{k}\right\|_{L^{\theta}(\gamma, s)\left(B_{1}\right)}\right]
$$

whence, by rescaling back to $B_{\rho}$ and by Lemma 2 of [19], we deduce

$$
\begin{aligned}
\left\|D u_{k}\right\|_{L\left(\frac{\theta \gamma}{\theta-\gamma}, \frac{\theta s}{\theta-\gamma}\right)\left(B_{\rho / n^{4}}\right) \leq} & c \rho^{(n-\theta) \frac{\theta-\gamma}{\theta \gamma}} \\
& \times\left[\left\|D u_{k}\right\|_{L^{1, \frac{\theta-\gamma}{\gamma}}\left(B_{9 / 10 \rho}\right)}+\left\|f_{k}\right\|_{L^{\theta}(\gamma, s)\left(B_{\rho}\right)}\right] .
\end{aligned}
$$

The covering argument of step 5 of [19] and (41) give

$$
\left\|D u_{k}\right\|_{L^{\theta}\left(\frac{\theta \gamma}{\theta-\gamma}, \frac{\theta s}{\theta-\gamma}\right)\left(B_{R / 2}\right)} \leq c\left[\left\|D u_{k}\right\|_{L^{1, \frac{\theta-\gamma}{\gamma}}\left(B_{27 / 40 R}\right)}+\left\|f_{k}\right\|_{L^{\theta}(\gamma, s)\left(B_{3 / 4 R}\right)}\right]
$$

for any $B_{R} \subset \subset \Omega$.

10 The proof of these steps remains unchanged in the case of several equations (i.e. $N \geq 2$ ).

${ }^{11} Q_{\text {inn }}\left(B_{1}\right)$ is the inner cube of $B_{1}$ as defined in footnote 7 . 
We now apply (33) on the right-hand side of (42) inferring

$$
\left\|D u_{k}\right\|_{L^{\theta}\left(\frac{\theta \gamma}{\theta-\gamma}, \frac{\theta s}{\theta-\gamma}\right)\left(B_{R / 2}\right)} \leq c R^{\frac{\theta-\gamma}{\gamma}-n}\left[\left\|D u_{k}\right\|_{L^{1}\left(B_{R}\right)}+\left\|f_{k}\right\|_{L^{\theta}(\gamma, s)\left(B_{R}\right)}\right]
$$

and we conclude the proof by passing to the limit in (43).

We now state some Theorems whose proofs can be deduced, by virtue of Theorem 5.1, arguing respectively as in the proofs of the Theorems 1, 4 and 3 from [19]. It is worthwhile to note that the proofs of the aforementioned theorems remain unchanged in case of several equations.

Theorem 5.2. Let $f \in L^{\gamma, \theta}\left(\Omega, \mathbb{R}^{N}\right)$, with $\left.\left.\theta \in\right] 2, n\right]$ and $\left.\left.\gamma \in\right] 1, \frac{2 \theta}{\theta+2}\right]$, and let $u \in W_{0}^{1,1}\left(\Omega, \mathbb{R}^{n N}\right)$ be the Stampacchia solution to the problem (1).

Then

$$
D u \in L^{\frac{\theta \gamma}{\theta-\gamma}, \theta}\left(\Omega, \mathbb{R}^{n N}\right)
$$

and there exists a positive constant $c \equiv c\left(n, \Lambda_{1}, \Lambda_{2}, \gamma\right)$ such that it holds

$$
\|D u\|_{L^{\frac{\theta \gamma}{\theta-\gamma}, \theta}\left(B_{R / 2}\right)} \leq c R^{\frac{\theta-\gamma}{\gamma}-n}\left[\|D u\|_{L^{1}\left(B_{R}\right)}+\|f\|_{L^{\gamma, \theta}\left(B_{R}\right)}\right]
$$

for every ball $B_{R} \subset \subset \Omega$.

Theorem 5.3. Let $f \in L^{\gamma, \theta}\left(\Omega, \mathbb{R}^{N}\right)$, with $\theta \in[2, n]$ and $\gamma>\frac{2 \theta}{\theta+2}$, and let $u \in W_{0}^{1,1}\left(\Omega, \mathbb{R}^{n N}\right)$ be the Stampacchia solution to the problem (1).

Then

$$
D u \in L^{h, \theta}\left(\Omega, \mathbb{R}^{n N}\right)
$$

for some $h \equiv h\left(n, \Lambda_{1}, \Lambda_{2}, \gamma, \theta\right)>2$ and there exists a positive constant $c \equiv$ $c\left(n, \Lambda_{1}, \Lambda_{2}\right)$ such that it holds

$$
\|D u\|_{L^{h, \theta}\left(B_{R / 2}\right)} \leq c R^{\frac{\theta}{h}-n}\left[\|D u\|_{L^{1}\left(B_{R}\right)}+\|f\|_{L^{\gamma, \theta}\left(B_{R}\right)}\right]
$$

for every ball $B_{R} \subset \subset \Omega$.

Theorem 5.4. Let $f \in L^{1, \theta} \cap L \log L\left(\Omega, \mathbb{R}^{N}\right)$, with $\theta \in[2, n]$ and let $u$ be the solution to the problem (1).

Then

$$
D u \in L^{\frac{\theta}{\theta-1}}\left(\Omega, \mathbb{R}^{n N}\right)
$$

and there exists a positive constant $c \equiv c\left(n, \Lambda_{1}, \Lambda_{2}\right)$ such that it holds

$$
\begin{aligned}
& \left(\int_{B_{R / 2}}|D u|^{\frac{\theta}{\theta-1}} d x\right)^{\frac{\theta}{\theta-1}} \leq c f_{B_{R}}|D u| d x \\
& +c\|f\|_{L^{1, \theta}\left(B_{R}\right)}^{\frac{1}{\theta}}\left[f_{B_{R}}|f| \log \left(e+\frac{|f|}{f_{B_{R}}|f(y)| d y}\right) d x\right]^{\frac{\theta-1}{\theta}}
\end{aligned}
$$

for every ball $B_{R} \subset \subset \Omega$. 
Theorem 5.5. Let $f \in L(\gamma, s)\left(\Omega, \mathbb{R}^{N}\right)$, with $\left.\left.\gamma \in\right] 1, \frac{2 n}{n+2}\right]$ and $\left.\left.s \in\right] 0,+\infty\right]$, and let $u \in W_{0}^{1,1}\left(\Omega, R^{n N}\right)$ be the solution to the problem (1).

Then

$$
D u \in L_{l o c}\left(\frac{n \gamma}{n-\gamma}, s\right)
$$

and there exists a positive constant $c \equiv c\left(n, \Lambda_{1}, \Lambda_{2}, \gamma, s\right)$ such that it holds

$$
\|D u\|_{L\left(\frac{n \gamma}{n-\gamma}, s\right)\left(B_{R / 2}\right)} \leq c\left[R^{\frac{n-\gamma}{\gamma}-n}\|D u\|_{L^{1}\left(B_{R}\right)}+\|f\|_{L(\gamma, s)}\left(B_{R}\right)\right] .
$$

for every ball $B_{R} \subset \subset \Omega$.

As a consequence we obtain the following

Corollary 5.1. Let $f \in L\left(\frac{2 n}{n+2}, s\right)\left(\Omega, \mathbb{R}^{N}\right)$ and let the rest of the assumptions of the Theorem be satisfied.

Then

$$
D u \in L_{l o c}(2, s)\left(\Omega, \mathbb{R}^{n N}\right) .
$$

\section{Integrability of $u$}

We assume that conditions (3) and (4) be satisfied and we start with the following Lemma analog to Lemma 10 from [19].

Lemma 6.1. Let $u \in W_{0}^{1,2}\left(\Omega, \mathbb{R}^{N}\right)$ be the solution to the problem (20).

Then there exists an absolute constant $C \equiv C\left(n, \Lambda_{1}, \Lambda_{2}\right)>1$ such that: for every $T>1$ and $\chi_{0}>1$, there exists a positive constant $\varepsilon \equiv \varepsilon\left(n, \Lambda_{1}, \Lambda_{2}, T\right.$, $\left.\left.\chi_{0}\right) \in\right] 0,1\left[\right.$ such that, if $\lambda>0$ and $Q$ is a dyadic sub-cube of $Q_{0}$ such that

$$
\left|Q \cap\left\{x \in Q_{0}: M^{*}(|u|)(x)>C T \lambda, M_{2}^{*}(f)(x) \leq \varepsilon \lambda\right\}\right|>T^{-2 \chi_{0}}|Q|,
$$

then its predecessor $\tilde{Q}$ satisfies

$$
\tilde{Q} \subseteq\left\{x \in Q_{0}: M^{*}(|u|)(x)>\lambda\right\} .
$$

Proof. The proof proceeds as the one of Lemma 4.2 with some modifications.

Indeed, we start again by contradiction and we obtain the following ana$\log$ of inequality (27)

$$
M_{2}^{*}(f)(\bar{x}) \leq \varepsilon \lambda
$$

for some $\bar{x} \in Q$. In turn, inequality (29) is replaced by

$$
\left|B_{R}\right|^{2 / n} f_{B_{R}}|f| d x \leq c(n) \varepsilon \lambda, \quad R \leq 1 .
$$

By virtue of Poincaré inequality and (23) we get the analog of inequality (30)

$$
f_{3 Q}|u-v| d x \leq c_{V}\left(n, \Lambda_{1}, \Lambda_{2}\right)\left|B_{R}\right|^{2 / n} f_{B_{R}}|f| d x \leq c \varepsilon \lambda .
$$


Using (49) and (19) we obtain

$$
f_{2 Q}|v|^{2 \chi_{0}} d x \leq c \lambda^{2 \chi_{0}}
$$

which replaces (32) and the proof can be completed as in Lemma 12 of [19].

From the previous Lemma, following the proof of Theorems 14 and 15 from [19] and Theorem 5.1 one can prove the next two Theorems.

Theorem 6.1. Let $f \in L^{\theta}(\gamma, s)\left(\Omega, \mathbb{R}^{N}\right)$, with $\left.\left.\left.\left.\gamma \in\right] 1, \theta / 2[, \theta \in] 2, n\right], s \in\right] 0,+\infty\right]$, and let $u \in W_{0}^{1,1}\left(\Omega, \mathbb{R}^{n N}\right)$ be the solution to the problem (1).

Then

$$
u \in L_{l o c}^{\theta}\left(\frac{\theta \gamma}{\theta-2 \gamma}, \frac{\theta s}{\theta-2 \gamma}\right)\left(\Omega, \mathbb{R}^{N}\right)
$$

and there exists a positive constant $c \equiv c_{V}\left(n, \lambda_{1}, \Lambda_{2}, \gamma, \theta, s\right)$ such that it holds

$$
\|u\|_{L^{\theta}\left(\frac{\theta \gamma}{\theta-2 \gamma}, \frac{\theta s}{\theta-2 \gamma}\right)\left(B_{R / 2}\right)} \leq c\left[R^{\frac{\theta-2 \gamma}{\gamma}-n}\|u\|_{L^{1}\left(B_{R}\right)}+\|f\|_{L^{\theta}(\gamma, s)\left(B_{R}\right)}\right]
$$

for every ball $B_{R} \subset \subset \Omega$.

Theorem 6.2. Let $f \in L(\gamma, s)\left(\Omega, \mathbb{R}^{N}\right)$, with $\left.\left.\gamma \in\right] 1, n / 2[, s \in] 0,+\infty\right]$, and let $u \in W_{0}^{1,1}\left(\Omega, \mathbb{R}^{n N}\right)$ be the solution to the problem $(1)$.

Then

$$
u \in L_{l o c}\left(\frac{n \gamma}{n-2 \gamma}, s\right)\left(\Omega, \mathbb{R}^{N}\right)
$$

and there exists a positive constant $c \equiv c_{V}\left(n, \lambda_{1}, \Lambda_{2}, \gamma, s\right)$ such that it holds

$$
\|u\|_{L\left(\frac{n \gamma}{n-2 \gamma}, s\right)\left(B_{R / 2}\right)} \leq c\left[R^{\frac{n-2 \gamma}{\gamma}-n}\|u\|_{L^{1}\left(B_{R}\right)}+\|f\|_{L(\gamma, s)\left(B_{R}\right)}\right]
$$

for every ball $B_{R} \subset \subset \Omega$.

Finally, we prove the following Theorem which deals with a borderline case.

Theorem 6.3. Let $f \in \mathcal{M}^{\theta / 2, \theta}\left(\Omega, \mathbb{R}^{N}\right)$, with $\left.\left.\theta \in\right] 2, n\right]$, and let $u \in W_{0}^{1,1}\left(\Omega, \mathbb{R}^{N}\right)$ be the solution to the problem (1).

Then

$$
u \in B M O_{l o c}\left(\Omega, \mathbb{R}^{N}\right)
$$

and there exists a positive constant $c \equiv c_{V}\left(n, \Lambda_{1}, \Lambda_{2}, \theta\right)$ such that it holds

$$
[u]_{B M O\left(B_{R / 2}\right)} \leq c\left[R^{1-n}\|D u\|_{L^{1}\left(B_{R}\right)}+\|f\|_{\mathcal{M}^{\theta / 2, \theta}\left(B_{R}\right)}\right]
$$

for every ball $B_{R} \subset \subset \Omega$.

Proof. The proof of (53) follows via Lemma 4.3 and the fact that $B M O \equiv$ $\mathcal{L}^{1, n} \cdot{ }^{12}$

12 Inequality (53) can be proved also as in the Corollary 6.2 of [17]. 


\section{Differentiability of $\mathrm{Du}$}

In this Section we will assume that the coefficients $A_{i j}$ are smoother than the previous Sections, namely we assume condition (3) and we suppose that

$$
\left.\left.A_{i j} \in C^{0, \alpha}\left(\Omega, \mathbb{R}^{N^{2}}\right) \text { for some } \alpha \in\right] 0,1\right] \text {. }
$$

Following the idea of [19] we prove the following

Theorem 7.1. Let $f \in L^{\gamma, \theta}\left(\Omega, \mathbb{R}^{N}\right)$, with $\left.\left.\gamma \in\right] 1, \frac{2 \theta}{\theta+2}\right]$ and $\left.\left.\theta \in\right] 2, n\right]$, and let $u \in W_{0}^{1,1}\left(\Omega, \mathbb{R}^{n N}\right)$ be the solution to the problem $(1)$.

Then, for every $\sigma<1$,

$$
D u \in W_{l o c}^{\alpha \sigma, \gamma, \theta}\left(\Omega, \mathbb{R}^{n N}\right)
$$

and there exists a positive constant $c \equiv c\left(n, \Lambda_{1}, \Lambda_{2}, \alpha, \gamma, \theta, \sigma,\left[A_{i j}\right]_{C^{0, \alpha}}\right)$ such that it holds

$$
[D u]_{W^{\alpha \sigma, \gamma, \theta}\left(B_{R / 2}\right)} \leq c\left[R^{\frac{\theta-n \gamma}{\gamma}-\alpha \sigma}\|D u\|_{L^{1}\left(B_{R}\right)}+R^{-\alpha \sigma}\|R f\|_{L^{\gamma, \theta}\left(B_{R}\right)}\right]
$$

for every ball $B_{R} \subset \subset \Omega$.

Proof. We will follow the idea of Lemmata 4.4 and 4.5 from [12] for the approximating solution $u\left(\equiv u_{k}\right)$ and then we pass to the limit.

Instead of (23) from [12] we set

$$
\left\{\begin{array}{l}
q=\gamma \\
\delta=1 \\
\gamma_{\alpha}(t)=\frac{\alpha}{\alpha+1-t} \quad \text { for every } t \in[0,1+\alpha[
\end{array}\right.
$$

we fix a ball $B_{R} \subset \subset \Omega$ and we set $\hat{B}=B_{16 R} \subset \subset \Omega$

With the aforementioned notation, Lemma 4.4 from [12] works replacing everywhere $|\bar{f}|$ by $|f|^{\gamma}$ provided we use (13) of Corollary 2.1. Specifically, estimate (33) from [12] is replaced by

$$
\int_{\hat{B}}|D u-D v|^{\gamma} d x \leq c\left\||f|^{\gamma}\right\|_{L^{1}(\hat{B})}|h|^{\beta \gamma} \cdot{ }^{13}
$$

The rest of the proof remains unchanged and thus the conclusion is that the implication

$$
\begin{array}{ll}
D u \in W_{l o c}^{t, \gamma}\left(\Omega, \mathbb{R}^{n N}\right) & \text { for some } t \in[0, \alpha[\Rightarrow \\
D u \in W_{l o c}^{\tilde{t}, \gamma}\left(\Omega, \mathbb{R}^{n N}\right) & \forall \tilde{t} \in\left[0, \gamma_{\alpha}(t)[\right.
\end{array}
$$

holds, that is the implication

$$
D u \in W_{l o c}^{t, \gamma}\left(\Omega, \mathbb{R}^{n N}\right) \Rightarrow D u \in W_{l o c}^{\tilde{t}, \gamma}\left(\Omega, \mathbb{R}^{n N}\right)
$$

holds for every $t<\alpha$ whenever $\tilde{t} \in\left[t, \gamma_{\alpha}(t)[\right.$.

$\overline{13 \text { Recall that } 0}<|h|<<1$ and $\beta=\frac{1}{1-t-\alpha}$. 
Along with (57) we have that for every couple of open subsets $\Omega^{\prime} \subset \subset$ $\Omega^{\prime \prime} \subset \subset \Omega$ there exists a positive constant $c=c\left(n, \frac{\Lambda_{1}}{\Lambda_{2}}, q, d\left(\Omega^{\prime}, \partial \Omega^{\prime \prime}\right), \tilde{t},\left[A_{i j}\right]_{C^{0, \alpha}}\right)$ such that

$$
[D u]_{W^{\tilde{t}, \gamma}\left(\Omega^{\prime}, \mathbb{R}^{n N}\right)}^{\gamma} \leq c \int_{\Omega^{\prime \prime}}\left(|D u|^{\gamma}+\left|f^{\gamma}\right|\right) d x .
$$

The implication (57) is the starting point of an iterative procedure like the one of Lemma 6.2 of [18] (according to the notation used in Lemma 4.5 of $[12])$. The final outcome is that

$$
D u \in W_{l o c}^{t, \gamma}\left(\Omega, \mathbb{R}^{n N}\right) \quad \text { for every } \quad t \in[0, \alpha[
$$

which implies the first part of the thesis.

The inequality (54) now follows as in the proof of Theorem 5 from [19] also taking into account inequality (44) of Theorem 5.2.

\section{Acknowledgments}

The author thanks G. Mingione for stimulating discussions.

\section{References}

[1] Acosta, G., Durán, R.G.: An optimal Poincaré inequality in $L^{1}$ for convex domains. Proc. Am. Math. Soc. 132, 195-202 (2004)

[2] Acquistapace, P.: On BMO regularity for linear elliptic systems. Ann. Mat. Pura e App. IV CLXI, 231-270 (1992)

[3] Adams, D.R.: A note on Riesz potentials. Duke Math. J. 42, 765-778 (1975)

[4] Boccardo, L., Gallouët, T.: Nonlinear elliptic and parabolic equations involving measure data. J. Funct. Anal. 87(1), 149-169 (1989)

[5] Boccardo, L., Gallouët, T.: Nonlinear elliptic equations with right-hand side measures. Commun. Partial Differ. Equ. 17, 641-655 (1992)

[6] Campanato, S.: Proprietà di inclusione per spazi di Morrey. Ricerche Mat. 12, 67-86 (1963)

[7] Campanato, S.: Proprietà di una famiglia di spazi funzionali. Ann. Scuola Nor. Sup. Pisa Cl. Sci. 3 18, 137-160 (1964)

[8] Campanato, S.: Sistemi Ellittici in Forma Divergenza. Regolarità all'Interno. Quaderni Scuola Normale Superiore Pisa, Pisa (1980)

[9] Chiarenza, F.: $L^{p}$-regularity of PDE's with coefficients in VMO. In: Nonlinear Analysis, Function Spaces and Applications, vol. 5. Proceedings of the Spring School held in Prague. Prometheus Publishing House (1994) 
[10] Cirmi, G.R., Leonardi, S.: Regularity results for the gradient of solutions of linear elliptic equations with $L^{1, \lambda}$ data. Annali di Mat. Pura E Appl. (4) 185, 537-553 (2006)

[11] Cirmi, G.R., Leonardi, S.: Regularity results for the gradient of nonlinear elliptic equations with $L^{1, \lambda}$ data. Nonlinear Anal. T.M.A. 69(1), 230-244 (2008)

[12] Cirmi, G.R., Leonardi, S.: Higher differentiability for solutions of linear elliptic systems with measure data. Discr. Contin. Dyn. Syst. 26(1), 89-104 (2010)

[13] Cirmi, G.R., Leonardi, S., Stará, J.: Regularity results for the gradient of solutions of a class of linear elliptic systems with $L^{1, \lambda}$ data. Nonlinear Anal. T.M.A. 68(12), 3609-3624 (2008)

[14] De Giorgi, E.: Un esempio di estremali discontinue per un problema variazionale di tipo ellittico. Boll. Unione Mat. Italiana 4, 607-627 (1968)

[15] Kristensen, J., Mingione, G.: The singular set of minima of integral functionals. Arch. Ration. Mech. Anal. 180, 331-398 (2006)

[16] Leonardi, S., Kottas, J., Stará, J.: Hölder regularity of the solutions of some classes of elliptic systems in convex non smooth domains. Nonlinear Anal. T.M.A. 60(5), 925-944 (2005)

[17] Leonardi, S., Stará, J.: Regularity results for the gradient of solutions of linear elliptic systems with VMO-coefficients and $L^{1, \lambda}$ data. Forum Math. 22(5), 913$940(2010)$

[18] Mingione, G.: The Calderón-Zygmund theory for elliptic problems with measure data. Ann. Scuola Norm. Sup. Pisa Cl. Sci. (5) VI, 195-261 (2007)

[19] Mingione, G.: Gradient estimates below the duality exponent. Math. Ann. 346(3), 571-627 (2010)

[20] Sarason, D.: Functions with vanishing mean oscillations. Trans. A.M.S., 207, (1975)

[21] Stampacchia, G.: The spaces $L^{(p, \lambda)}, N^{(p, \lambda)}$ and interpolation. Ann. Scu. Norm. Sup. Pisa Cl. Sci. (III) 19, 443-462 (1965)

S. Leonardi

Dipartimento di Matematica ed Informatica,

Università di Catania,

Viale A. Doria 6,

95125 Catania,

Italy

e-mail: leonardi@dmi.unict.it

Received: 03 May 2010.

Revised: 07 September 2010.

Accepted: 12 November 2010. 